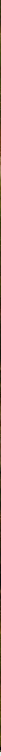

\title{
Ciencias de la atmósfera
}

Marcos Gómez Molina y Álvaro Subías díaz-Blanco

Área de Técnicas y Aplicaciones de Predicción (ATAP), Agencia Estatal de Meteorología (AEMET)

La ciencia es respecto del alma lo que es la luz respecto de los ojos, y si las raíces son amargas, los frutos son muy dulces.

ARISTÓTELES

Ofrecemos en este capítulo una introducción a las ciencias atmosféricas que puede servir como base para entender otros capítulos de este libro. En particular, el capítulo que versa sobre modelos atmosféricos puede ser opaco para el profano pero, con ayuda de esta introducción, puede abordarse su lectura y aumentar el grado de comprensión. Una introducción a las ciencias atmosféricas preparada para profanos es un auténtico reto. Intentar ahorrar en expresiones matemáticas, exprimir materia hasta quedarse en el concepto, equilibrar la completitud con la profundidad, sabiendo lo difícil que resulta todo ello. El abanico de las ciencias atmosféricas se va ampliando progresivamente y, a día de hoy, disciplinas como la química atmosférica resultan de vital importancia. Hemos querido ofrecer un capítulo asequible sin exceder un cierto número de páginas, por lo que hemos decidido quedarnos, si no con lo más esencial, con lo más clásico: la radiación, la termodinámica y la dinámica atmosféricas. Invitamos al lector interesado a acudir a textos especializados para profundizar en estas materias o bien para ampliar ese abanico tan difícil de abarcar.

Palabras clave: ciencias de la atmósfera, termodinámica atmosférica, radiación en la atmósfera, dinámica atmosférica. 


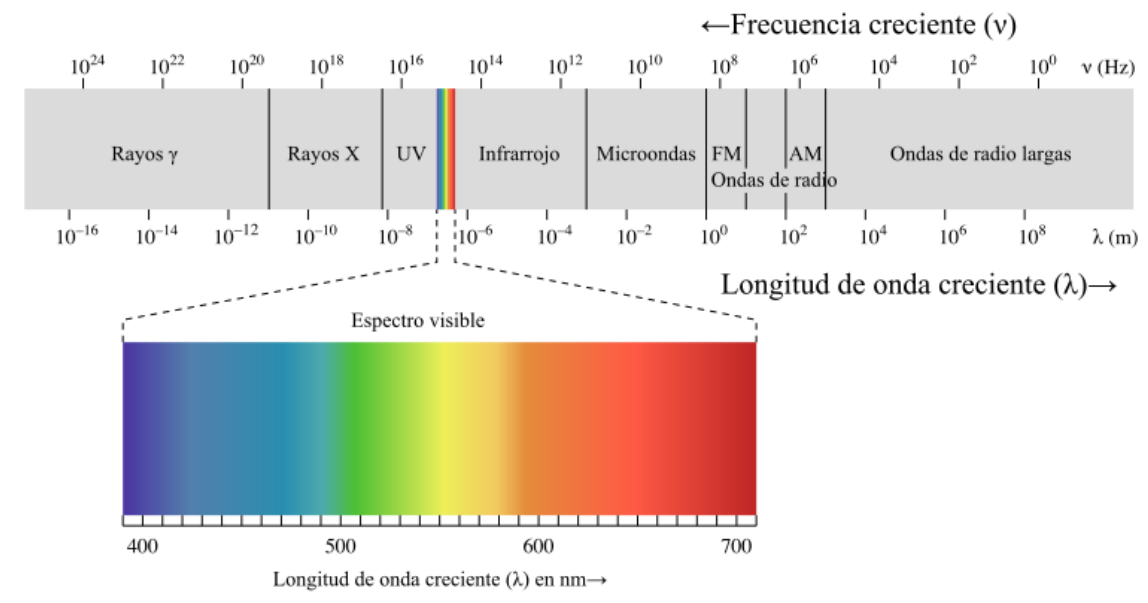

Figura 6.1: Espectro de radiación de las ondas electromagnéticas (ver texto) (PHILIP RONAN, CC-BY-SA https: //es.wikipedia. org/wiki/Archivo: EM_spectrum_es. svg).

\subsection{Radiación}

La radiación solar es la responsable del movimiento de aire en la atmósfera y por lo tanto de los fenómenos asociados que conocemos habitualmente como $\mathrm{el}$ tiempo. Por tanto, comprender la interacción entre la radiación y el sistema Tierra-atmósfera es el primer paso para comprender su funcionamiento.

Lo primero que hay que destacar es que la radiación solar supone más del $99 \%$ de la energía que entra en el sistema y por tanto, a efectos prácticos, se considera su única fuente de energía. Pero lo realmente importante de toda esta energía es la forma en la que se distribuye. Por lo pronto diremos que no se distribuye de forma homogénea lo cual crea un calentamiento desigual que atmósfera y océanos tratan incesantemente de compensar para alcanzar así el equilibrio térmico. Los movimientos que se producen en el sistema Tierra-atmósfera tratan por tanto de llevar calor de las zonas más calientes a las más frías.

\subsubsection{Leyes y conceptos fundamentales}

Cuando hablamos de radiación electromagnética es habitual pensar en la luz solar que podemos ver y que como sabemos está compuesta por multitud de colores como queda de manifiesto al ver el arco iris. Podemos decir que la luz está caracterizada por su color. Si extendemos este concepto al resto de ondas electromagnéticas decimos que la radiación está caracterizada por su energía, o lo que es lo mismo por su longitud de onda.
El estudio de la materia y su interacción con las diferentes longitudes de onda de la radiación se llama espectroscopía. Así, cuando hablamos de espectro de un cuerpo o compuesto nos referimos al análisis de su radiación emitida o absorbida. Cada compuesto se caracteriza por su espectro de absorción y de emisión, esto es algo así como una huella dactilar que lo identifica. Que cada elemento o compuesto químico tenga un espectro diferente es importante ya que permite determinar la composición de un cuerpo a partir del análisis de su radiación.

En la Figura 6.1 podemos ver todo el espectro electromagnético, del cual a efectos prácticos solo nos interesa la radiación ultravioleta, visible e infrarroja. Nótese lo estrecha que es la franja visible en comparación al resto.

Por último, veamos de forma cualitativa las leyes y conceptos más importantes que gobiernan la radiación:

- Todos los cuerpos emiten radiación por el hecho de encontrarse a cierta temperatura. Por tanto no solo el Sol emite radiación sino que el suelo, las nubes, el agua, el hielo también lo hacen. El caso del Sol o de una bombilla es más evidente porque parte de la radiación es visible a nuestros ojos. Esto se conoce como Ley de PLANCK, y a partir de ella se deducen las dos leyes siguientes.

- Los cuerpos más calientes emiten más energía que los más fríos, según la Ley de STEFANBOLTZMANN.

- Cuanto más caliente esté un cuerpo menor será la longitud de onda de la radiación máxima que emite, según la Ley de WIEN. 
- Los cuerpos que emiten bien la radiación también la absorben bien. Esto se traduce en que los espectros de absorción y de emisión son complementarios.

\subsubsection{La radiación solar y su espectro electromagnético}

El espectro del Sol, es decir la cantidad de energía en función de la longitud de onda puede verse en la Figura 6.2. Fijémonos en primer lugar en el contorno amarillo que muestra la radiación solar antes de entrar en la atmósfera. La línea negra indica la radiación que predice la Ley de PLANCK para un cuerpo a $5250^{\circ} \mathrm{C}$. Nótese lo bien que ajustan ambas gráficas. Según dicha ley podemos identificar esa temperatura como la de la superficie del Sol.

Aunque la parte visible parece un región estrecha en la gráfica, al corresponderse con el máximo, supone el $49 \%$ de la energía total. A la izquierda de la misma, en las longitudes de onda más cortas queda la radiación ultravioleta con un $9 \%$ de la energía, responsable de ponernos morenos y de las quemaduras que nos produce un larga exposición al Sol. La radiación infrarroja queda a la derecha con un $42 \%$ de la energía. Nuestros ojos no la pueden apreciar pero sí la podemos sentir, por ejemplo acercando nuestra mano a algo caliente como es nuestro propio cuerpo.

El área roja se corresponde con la radiación que ha logrado traspasar la atmósfera y llega al suelo. Queda por ver lo que ocurre con la energía de la región amarilla. El estudio de la interacción radiación-materia muestra la clave en esta cuestión.

\subsubsection{Calentamiento desigual}

Veamos ahora las causas principales que crean diferencias de temperatura. Ejemplos de calentamiento desigual los tenemos todos los días, para empezar pensar simplemente en la diferencia de insolación de un día con cielo despejado a uno cubierto o en la diferencia de temperatura de un suelo de asfalto a uno de hierba. Estas diferencias de temperatura se deben a las diferentes características de los materiales a la hora de reflejar o absorber la radiación solar. Por otro lado, cabe pensar en las grandes diferencias entre verano e invierno. La diferencia de insolación estacional es debida a la inclinación que tiene el eje de la Tierra con respecto al plano de la órbita.

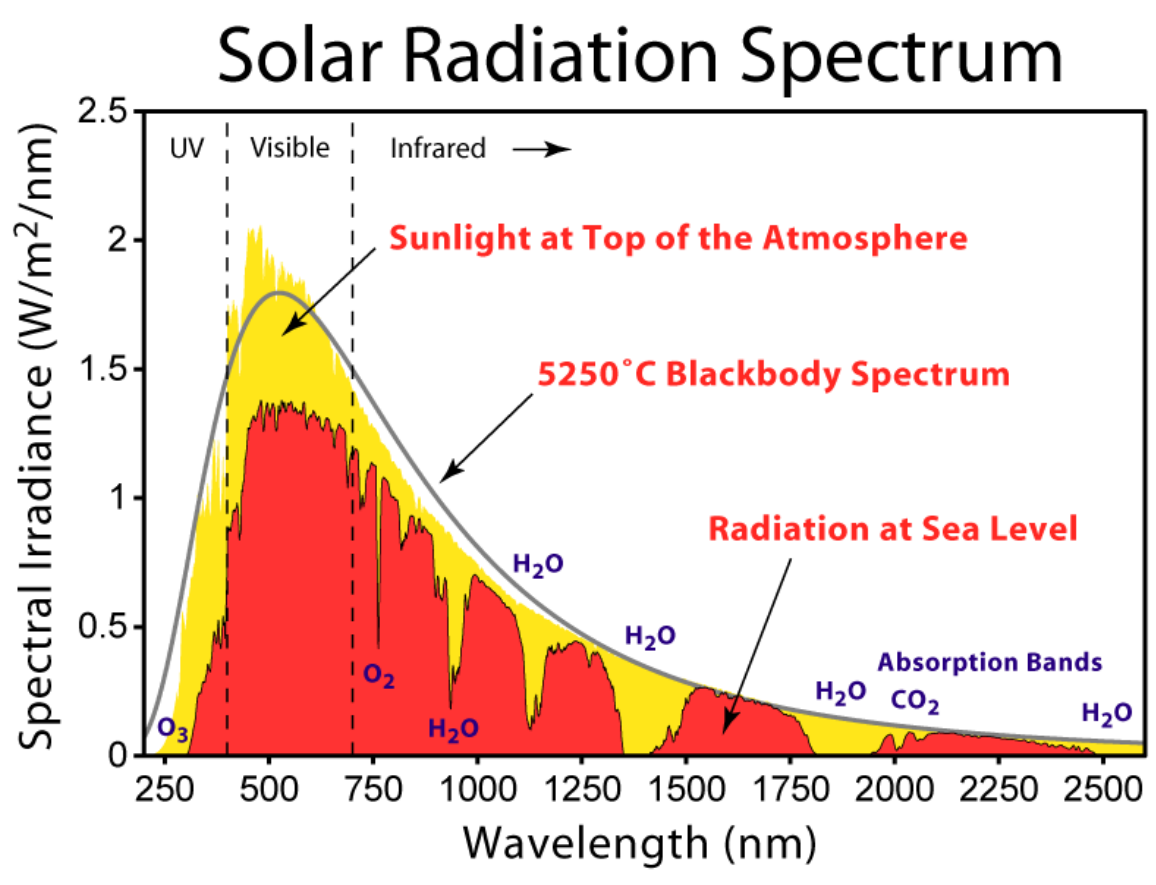

Figura 6.2: Espectro de la radiación solar por encima de la atmósfera (amarillo) y a nivel del mar (rojo) junto a la curva de emisión de cuerpo negro a $5250{ }^{\circ} \mathrm{C}$ (ver texto) (ROBERT A. ROHDE, GFDL, https: // commons . wikimedia. org/wiki/File: Solar_ Spectrum. png). 
Piénsese, por último, en la diferencia entre el ecuador, donde el Sol incide de forma casi perpendicular y los polos, donde lo hace de forma inclinada. La forma esférica de la tierra hace que el rayo de Sol esté más concentrado en torno al ecuador, aunque ilumine más área cerca de los polos. Este desequilibrio debido a la forma esférica de la tierra es el que provoca las mayores diferencias de calentamiento. Para compensarlo la atmósfera y océanos transportan calor constantemente desde el ecuador a los polos. Estas tres causas son suficientes para entender de forma cualitativa la desigualdad en el calentamiento que experimenta el sistema y que es responsable directo del movimiento atmosférico.

\subsubsection{Interacción radiación-materia}

La interacción radiación-materia nos permite comprender la cantidad de energía que es absorbida y crea por tanto el calentamiento diferencial del que hablábamos antes. Aunque la atmósfera es bastante transparente a la radiación del Sol, solo un $25 \%$ llega al suelo sin sufrir ninguna interacción. El resto será o bien absorbida por los gases que componen la atmósfera, o bien dispersada hasta que alcance la superficie o regrese al espacio, o reflejada directamente hacia el espacio. ¿De qué depende que sufra uno u otro proceso? Pues en gran medida de la longitud de onda de la radiación y del tamaño de las partículas con las que interacciona como veremos a continuación.

A modo de ejemplo podemos acompañar a un rayo de Sol a lo largo de su entrada en la atmósfera para ver qué tipo de interacciones sufre. Pues bien, puede ocurrir que nuestro rayo sea dispersado, es decir, que cambie su dirección pero no su energía. Esto ocurre al interaccionar con los gases, agua y aerosoles de la atmósfera. Este proceso depende de la energía de nuestro rayo y del tamaño de las partículas con las que interaccione. Las moléculas gaseosas dispersan de forma más efectiva los rayos más energéticos, lo que explica por qué vemos el cielo azul y por qué vemos el amanecer y anochecer de colores cálidos (dispersión de RAYLEIGH).

Las partículas más grandes, aerosoles y gotitas de agua, dispersan la luz de forma homogénea y por ello por ejemplo vemos las nubes blancas, todos los colores han sido dispersados por igual y no predomina ninguno de ellos. El color del cielo nos da idea de la cantidad de partículas de gran tamaño que hay presentes, cuando más azul es, más limpio está el aire.

También puede ocurrir que el rayo sea reflejado. Esto ocurre en la separación entre dos medios, por ejemplo entre el aire y el suelo o entre el aire y una gota de agua. Existen superficies que reflejan mejor que otras, la nieve, las nubes, y otras peor, el mar, la vegetación. El porcentaje de radiación solar que una superficie es capaz de reflejar se denomina albedo (Tabla 6.1), y varía mucho entre superficies. De valores inferiores al $5 \%$ en asfalto hasta valores del $90 \%$ en nieve recién caída. El albedo medio del sistema Tierra-atmósfera está en torno a 33\% (a lo cual contribuye también la dispersión que se produce hacia el espacio).

\begin{tabular}{|c|c|}
\hline Superficie & Albedo típico \\
\hline Asfalto (fresco-gastado) & 4 al 12\% \\
\hline Océano & $6 \%$ \\
\hline Bosque & 8 a 15\% \\
\hline Suelo desnudo & $17 \%$ \\
\hline Hierba verde & $25 \%$ \\
\hline Arena del desierto & $40 \%$ \\
\hline Hielo oceánico & 50 al 70\% \\
\hline Nieve fresca & 80 al 90\% \\
\hline
\end{tabular}

Tabla 6.1: Albedo promedio de diferentes medios (https: //es. wikipedia. org/wiki/Albedo).

Por último, nuestro rayo puede ser absorbido por los gases y partículas que componen la atmósfera o por el suelo. En el caso de los gases dependerá de la energía de nuestro rayo y del gas con el que interaccione. En general la atmósfera absorbe muy poco de luz visible y por eso llega hasta la superficie. Sin embargo la luz ultravioleta se absorbe casi completamente por el oxígeno y ozono, lo cual es de agradecer ya que tiene tremendos efectos sobre la salud. La radiación infrarroja interacciona fuertemente con el vapor de agua y dióxido de carbono. Como veremos a continuación esto tiene consecuencias muy importantes. Cuando un rayo es absorbido se incrementa la energía del átomo o molécula y esto se traduce en un incremento de temperatura del cuerpo.

Hay que hacer notar que nuestro rayo puede sufrir varias dispersiones y reflexiones en su viaje a través de la atmósfera y así puede que tras varios procesos logre escapar de nuevo hacia el espacio o, por el contrario, sea absorbido finalmente por la atmósfera o por el suelo. 


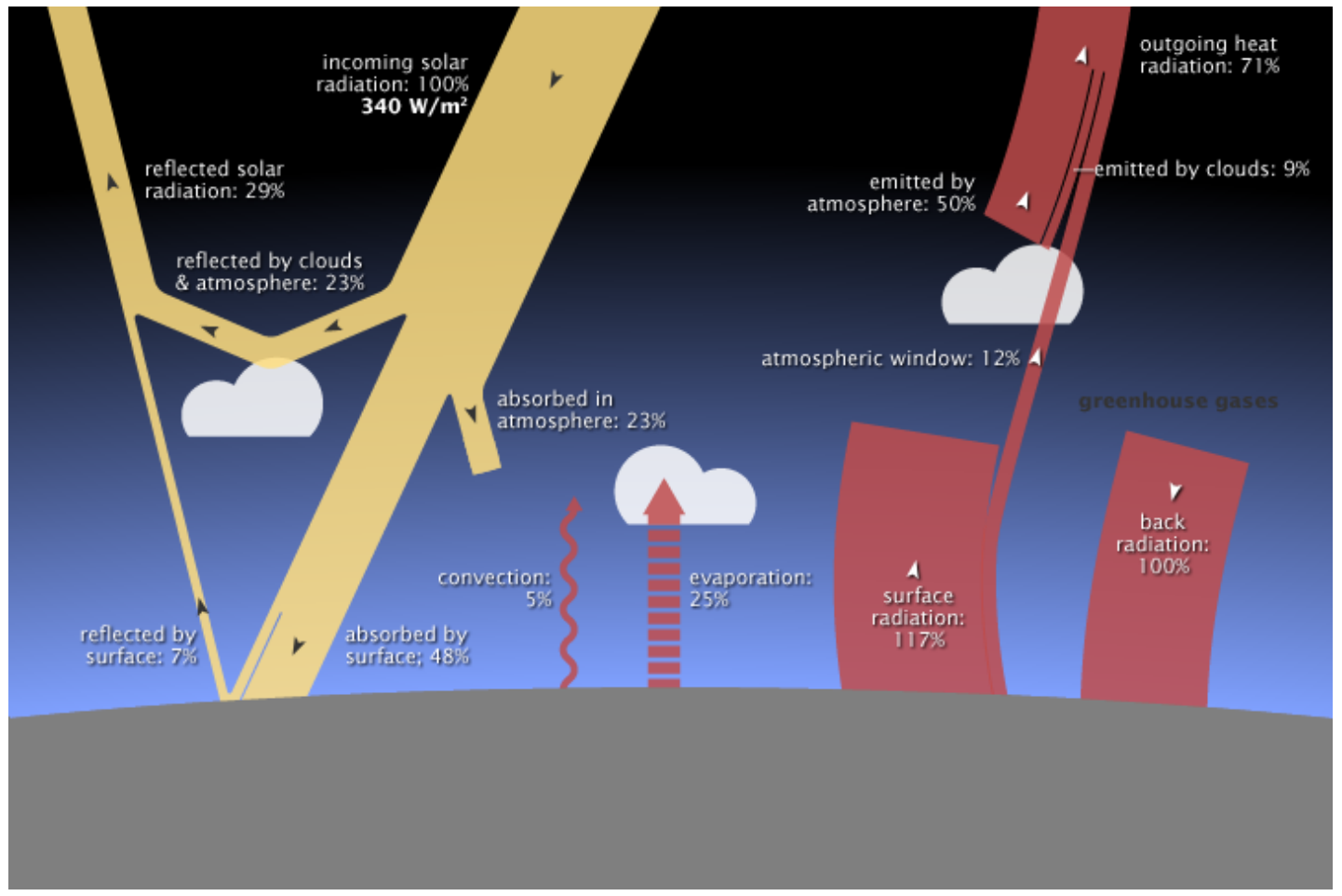

Figura 6.3: Balance de energía en la atmósfera (ver texto) (NASA, https://earthobservatory. nasa.gov/ ContentFeature/EnergyBalance/images/global_ energy_ budget_components. png ).

\subsubsection{Balance de energía}

La temperatura media de la Tierra, en periodos que no abarquen cambios climatológicos bruscos, se mantiene aproximadamente constante. Esto implica que el balance de energía en el sistema Tierra-atmósfera debe ser nulo y por tanto debe salir hacia el espacio la misma cantidad de energía que entra en el sistema. La salida de energía se produce en forma de radiación electromagnética. Un $29 \%$ de esta energía es radiación solar reflejada por las nubes y por el suelo, el resto, un $71 \%$, es energía en forma de radiación infrarroja. Como ya dijimos anteriormente, todo cuerpo emite radiación electromagnética por el simple hecho de estar a cierta temperatura. Con el suelo y los componentes de la atmósfera ocurre lo mismo, emiten radiación infrarroja que finalmente acaba saliendo al espacio y compensado de esta forma la radiación que entra. Pero cuidado, este proceso no es tan sencillo. Resulta que algunos gases como el $\mathrm{CO}_{2}$ y el vapor de agua son muy eficientes absorbiendo la radiación infrarroja. Esto implica que la radiación emitida por la tierra es en gran medida absorbida por los gases atmosféricos que a su vez vuelven a emitir parte de ella hacia el suelo. Se crea de esta forma un bucle o circuito que mantiene esta radiación infrarroja dentro de la atmósfera. Los gases responsables de este proceso se denominan de efecto invernadero y actúan de alguna forma como una manta que ayuda a mantener una temperatura más cálida en la Tierra, haciendo que gran parte de ella sea habitable. La Figura 6.3 esquematiza muy bien los principales efectos radiativos en un esquema vertical. Entiéndase que son valores promedio a escala global.

Hasta ahora hemos hablado sólo de radiación y su interacción con el sistema Tierra-atmósfera. Pero para acabar de comprender el balance de energía de este sistema hay que estudiar también la transferencia de calor que se produce por conducción y convección/advección. La conducción explica la transferencia de calor de un cuerpo más caliente a uno más frío cuando están en contacto, por ejemplo del suelo (más caliente) al aire (más frío) durante el día. La convección/advección es el transporte de calor que se produce por el movimiento de un volumen del aire de un sitio a otro. Hablamos de convección para movimientos verticales y advección para movimientos horizontales.

Hay que tener en cuenta que una buena parte del calor que llega al suelo se invierte en la evaporación de agua y que esa energía es liberada de nuevo en la atmósfera al producirse la condensación. Esta transferencia de energía se conoce como transferencia de calor latente y explica cómo un $25 \%$ de la energía es transportada desde el suelo hasta la atmósfera. 
Por último, decir que un análisis latitudinal de la radiación desvela que existe un gran desequilibrio en el balance de energía, existiendo déficit en los polos y superávit en el ecuador. Se deduce de este hecho que debe existir un transporte constante de energía desde el ecuador hacia los polos.

Para completar el capítulo resulta muy ilustrativo ver el efecto que producen los principales gases de la atmósfera en el espectro de radiación solar entrante y de radiación térmica saliente. Nótese que el vapor de agua es el principal gas de efecto invernadero y como la radiación infrarroja que logra escapar hacia el espacio (área azul) lo hace en una banda estrecha conocida como ventana atmosférica. Notar también como el oxígeno y ozono son responsables de absorber la mayor parte de la radiación ultravioleta.
A los espectros de radiación solar y terrestre se los denomina habitualmente como radiación de onda corta y de onda larga respectivamente. Las líneas continuas roja y azul se corresponden con los espectros teóricos dados por la ley de PLANCK para el Sol y la Tierra. La ley de Planck viene dada por:

$$
I(v, T)=\frac{2 h v^{3}}{c^{2}} \frac{1}{e^{\frac{h v}{k T}}-1}
$$

donde $I(v, T)$ es la radiancia espectral (cantidad de energía por unidad de superficie, unidad de tiempo y unidad de ángulo sólido por unidad de frecuencia o longitud de onda), $v$ es la frecuencia, $\lambda$ la longitud de onda, $T$ la temperatura del cuerpo negro, $h$ la constante de Planck, $c$ la velocidad de la luz y $k$ la constante de BoltzMann. Este efecto se ilustra en la Figura 6.4 .

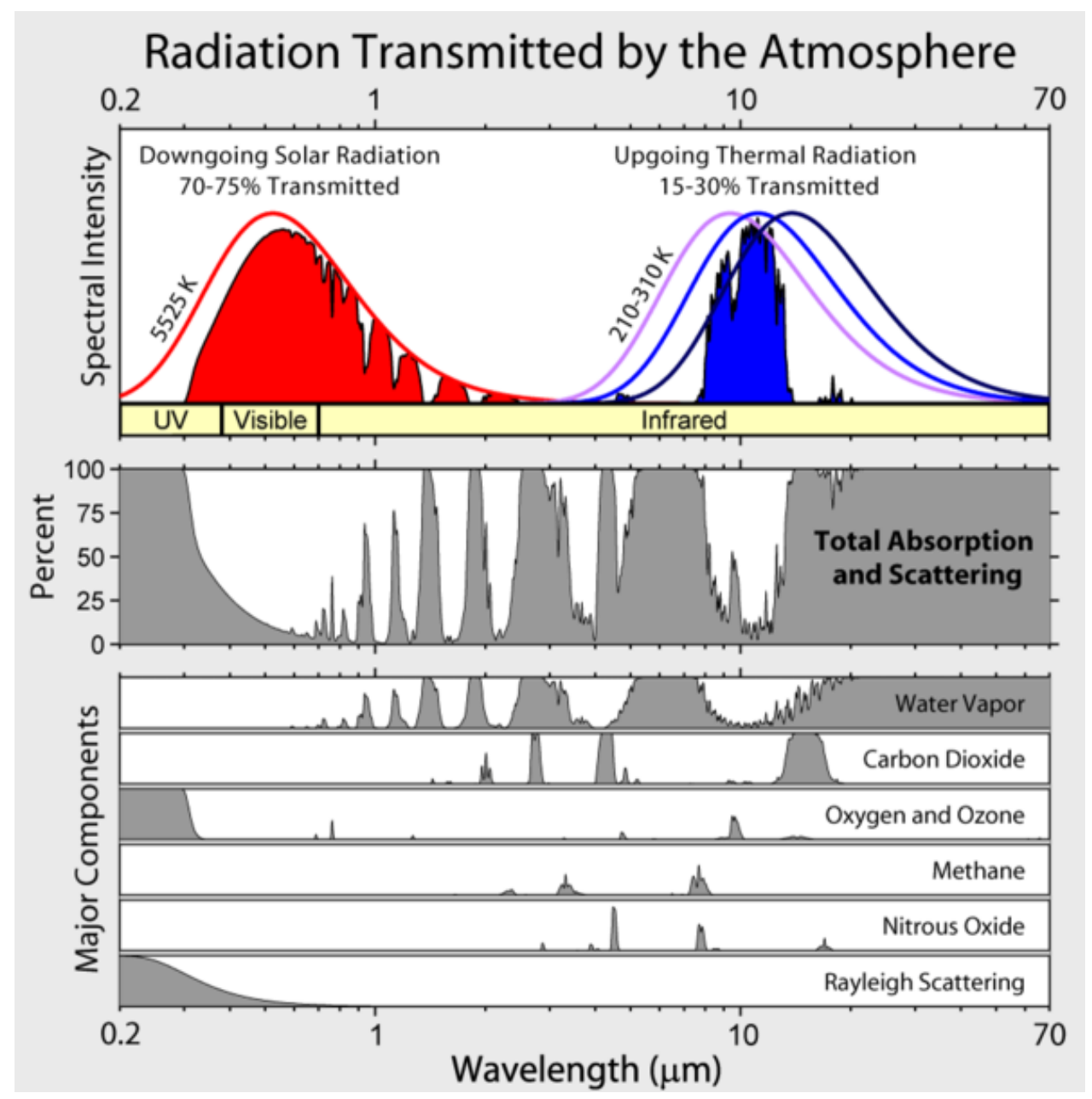

Figura 6.4: Bandas de absorción de los principales gases de efecto invernadero y su efecto sobre la radiación solar entrante y la radiación térmica saliente (ver texto) (ROBERT A. ROHDE, GFDL, https: // commons. wikimedia. org/wiki/File: Atmospheric_Transmission. png). 


\subsection{Termodinámica}

El propósito de este capítulo es explicar de forma cualitativa los fenómenos de naturaleza termodinámica de mayor interés. Para ello es necesario realizar una serie de simplificaciones y comprender las leyes que gobiernan el comportamiento de los gases y la termodinámica.

\subsubsection{Composición de la atmósfera}

La atmósfera está compuesta por: una mezcla de gases, agua en cualquiera de sus tres estados y partículas sólidas. En los primeros kilómetros, donde reside el interés meteorológico, la mezcla de gases es homogénea, y está compuesta de nitrógeno en un $78 \%$, oxígeno en un $21 \%$, argón en $0.9 \%$, dióxido de carbono en un $0.04 \%$, y el resto de componentes no alcanza el $0.003 \%$ en volumen. Algunos componentes como el $\mathrm{CO}_{2}$ y $\mathrm{O}_{3}$ presentan grandes variaciones de concentración pero de cara al estudio termodinámico son despreciables. Esta mezcla de gases, de la cual se excluye el vapor de agua, se denomina aire seco y en adelante se considerará como un único gas.

El agua presente en la atmósfera puede cambiar de estado y por la gran implicación que esto tiene merece un estudio detallado. Puede alcanzar valores de hasta un $4 \%$ pero presenta grandes variaciones, siendo máximo cerca del suelo y disminuyendo rápidamente con la altura. Esto es debido a que su fuente de entrada es la superficie terrestre a través de evaporación de océanos, lagos, ríos y transpiración de plantas y suelo, y a que la cantidad de vapor que puede contener el aire depende de la temperatura y por tanto decrece con la altura. Cuando el aire contiene cierta cantidad de vapor de agua se denomina aire húmedo.

Las partículas sólidas en suspensión, como la sal marina o el polvo mineral, se conoce como aerosoles atmosféricos. Son de gran importancia en física de nubes ya que son los núcleos de condensación en torno a los cuales comienzan a formarse las gotas de agua.

\subsubsection{Leyes y conceptos fundamentales}

Abordaremos la Ley de los gases ideales, el Primer principio de la termodinámica y el Segundo principio de la termodinámica.
La ley de los gases ideales es la ecuación de estado que describe el comportamiento de un gas ideal. La ventaja que presenta considerar un gas real como ideal es que simplifica su estudio en gran medida. El aire seco antes descrito puede tratarse como un gas ideal cometiendo un error inferior al 0,2\%. Considerar el vapor de agua como un gas ideal implica un mayor error pero en todo caso es asumible para gran parte de las aplicaciones meteorológicas y para el presente estudio.

La ecuación de estado de los gases ideales expresada en términos de la densidad $\rho$, la masa molecular $M$, la presión $P$ y la temperatura $T$ es:

$$
P=\rho \frac{R}{M} T
$$

Para conocer el estado de un gas ideal basta especificar dos de sus variables, típicamente $P$ y $T$.

Cuando dos sistemas están en contacto a diferente temperatura se produce una transferencia de energía en forma de calor, $Q$. Esta transferencia se produce hasta que los dos sistemas están a la misma temperatura. Los sistemas también pueden intercambiar energía en forma de trabajo, $W$. El ejemplo más intuitivo es pensar en trabajo mecánico, cuando una fuerza altera el movimiento de un cuerpo.

El primer principio nos dice que la variación de energía interna de un sistema se debe al calor y al trabajo intercambiado.

Primer principio de la termodinámica. En su forma matemática más sencilla, siguiendo el criterio de signos termodinámicos, se puede escribir para cualquier sistema cerrado como:

$$
\Delta U=Q+W
$$

$\Delta U$ es la variación de energía interna del sistema, $Q$ es el calor intercambiado por el sistema a través de unas paredes bien definidas, y $W$ es el trabajo intercambiado por el sistema con sus alrededores.

Este principio es un reflejo del principio de conservación de la energía aplicado al contexto de la termodinámica. En el caso más sencillo de un gas ideal la energía interna solo depende de la temperatura, lo que 
implica que un incremento de energía interna propicia un incremento de temperatura.

El segundo principio establece la asimetría que define los procesos irreversibles, distinguiéndolos de los reversibles.

Cuando ponemos en contacto dos cuerpos a diferente temperatura sabemos por experiencia que tienden al equilibrio térmico. No se nos ocurre pensar que el cuerpo frío cederá calor al más caliente para que siga aumentando su temperatura. Pues bien, esto mismo es lo que enuncia el segundo principio de la termodinámica, establece un criterio para saber si es posible que un proceso ocurra o no.

Segundo principio de la termodinámica. La formulación más clásica de este principio es:

$$
d S \geq \frac{\delta Q}{T}
$$

Donde $S$ es la función de estado llamada entropía. La igualdad se corresponde con procesos reversibles y la desigualdad con irreversibles o espontáneos.

\subsubsection{Procesos adiabáticos}

Se define proceso adiabático como aquel en que el sistema no intercambia calor con su entorno. Según el primer principio de la termodinámica esto implica que $\Delta U=W$. Muchos procesos que se dan en la atmósfera se pueden considerar muy aproximadamente como adiabáticos con lo cual sus cálculos se simplifican.

En termodinámica de la atmósfera se considera sistema a una determinada extensión de aire con unas propiedades homogéneas, típicamente de humedad y temperatura. Dependiendo del contexto se suele hablar de masa de aire para grandes extensiones a nivel sinóptico o de burbuja ${ }^{1}$ a nivel mesoescalar en el estudio de los movimientos verticales asociados a la convección. Las razones principales que justifican considerar el movimiento de una burbuja de aire como un proceso adiabático son:

- El aire es mal conductor del calor. Por tanto dos masas de aire a diferente temperatura tardarán un tiempo considerable en alcanzar el equilibrio térmico.

\footnotetext{
${ }^{1}$ parcela, término usual, es una traducción directa del término inglés parcel, que podría traducirse mejor tal vez como paquete o porción. En este capítulo mantendremos el término burbuja.

- La pared o región que delimita la masa de aire, donde se produce la mezcla con el exterior, es en general estrecha en comparación con la propia masa.

- Los procesos radiativos son pequeños comparados con los producidos por la expansión o compresión de los movimientos verticales, y más para periodos de tiempo inferiores a un día.

Gradiente adiabático seco. A partir del primer principio y considerando equilibrio hidrostático es posible calcular la variación de temperatura que sigue un proceso adiabático. El valor que se obtiene es:

$$
\gamma=0,98^{\circ} \mathrm{C} / 100 \mathrm{~m}
$$

A efectos mnemotécnicos basta quedarse con el valor de $1{ }^{\circ} \mathrm{C} / 100 \mathrm{~m}$ o $10^{\circ} \mathrm{C} / \mathrm{km}$.

Una burbuja de aire que asciende adiabáticamente se enfriará a razón de $1^{\circ} \mathrm{C}$ cada $100 \mathrm{~m}$, y se calentará dicha cantidad si desciende.

\subsubsection{Aire húmedo}

El aire húmedo consiste en la mezcla de aire seco y vapor de agua y puede tratarse como gas ideal. El error que se comente es inferior al $0.4 \%$ para los valores de presión y temperatura de interés. Ahora bien, dado que el vapor de agua tiene una masa molecular de $M_{v}=18,02 \mathrm{~g} / \mathrm{mol}$ y el aire seco de $M_{d}=28,96 \mathrm{~g} / \mathrm{mol}$, su mezcla dará como resultado un gas menos denso que el aire seco a igual presión y temperatura.

La humedad es la medida de la cantidad de vapor que contiene el aire. Es habitual expresar la cantidad de humedad del aire mediante la humedad relativa expresada en \% pero existen otras variables higrométricas de uso habitual en meteorología que conviene conocer:

- Humedad específica: $\frac{\text { masa de vapor }(\mathrm{g})}{\text { masa de aire humedo }(\mathrm{kg})}$

- Razón de mezcla: $\frac{\text { masa de vapor }(\mathrm{g})}{\text { masa de aire seco }(\mathrm{kg})}$. En la práctica puede considerarse igual a la humedad específica.

- Humedad relativa: $\frac{\text { masa de vapor }(\mathrm{g})}{\text { masa de vapor saturante }(\mathrm{g})} \times 100$. Es muy útil porque informa de lo alta que está la saturación.

- Temperatura virtual: Es la temperatura que tendría que tener el aire seco para tener la misma presión y densidad que el aire húmedo. 


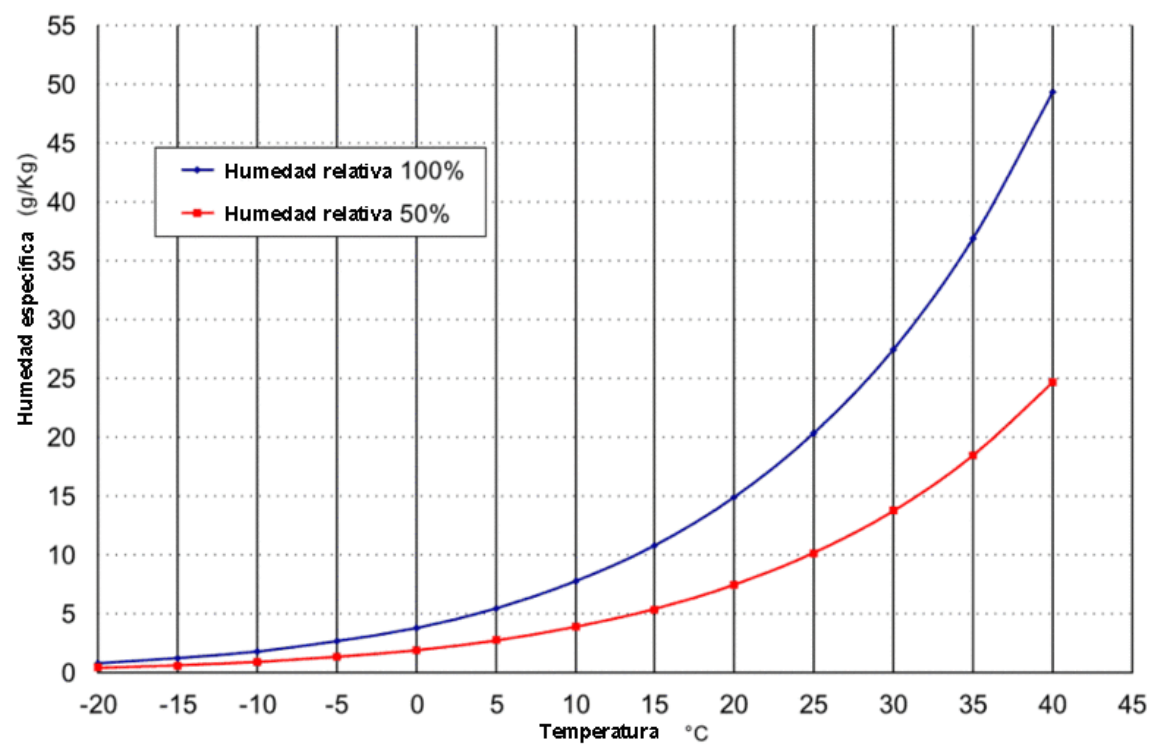

Figura 6.5: Humedad especifica en función de la temperatura a la presión de $1000 \mathrm{hPa}(\mathrm{CC}-\mathrm{BY}-\mathrm{SA}-3.0, \mathrm{ht}$ tps: // commons. wikimedia. org/wiki/File: Umidit\%C3\%AOspecifica. svg).

Cuando el aire húmedo no puede contener más vapor de agua se dice que está saturado. La cantidad de vapor de agua que puede contener el aire depende de la temperatura según la curva azul de la Figura 6.5.

Así para una temperatura de $10^{\circ} \mathrm{C}$ el aire puede contener $7,8 \mathrm{~g} / \mathrm{m}^{3}$ (humedad específica), su humedad relativa será del $100 \%$. Para $30^{\circ} \mathrm{C}$ el aire puede contener $27,3 \mathrm{~g} / \mathrm{m}^{3}$ y su humedad relativa será igualmente del $100 \%$.

Gradiente adiabático saturado. El aire húmedo sin saturar ante un proceso adiabático sigue muy aproximadamente el gradiente adiabático seco deducido anteriormente. Una vez se alcanza la saturación el agua condensada libera el calor latente que se ha invertido previamente para evaporarla. Este calor liberado contribuye a calentar el aire y a reducir el enfriamiento, por este motivo el gradiente adiabático saturado será siempre menor que el gradiente adiabático seco. La formulación del gradiente saturado es compleja y no tiene mayor importancia llegados a este punto, lo que si es de interés es dar algunos datos de referencia. El gradiente adiabático saturado oscila entre 4 y $7^{\circ} \mathrm{C} / \mathrm{km}$ en la troposfera media, mientras que el gradiente adiabático seco es de $10^{\circ} \mathrm{C} / \mathrm{km}$. A alturas elevadas el gradiente adiabático saturado se aproxima al seco.

Cuando el aire está saturado hay que considerar qué ocurre con el agua que comienza a condensar. Es habitual considerar que ese agua libera el calor latente que es absorbido completamente por el aire, y que el agua precipita inmediatamente. Esto implica una pérdida de entropía que favorece el enfriamiento y activa la condensación. Se conoce como proceso pseudoadiabático y en la práctica es la aproximación utilizada en meteorología.

\subsubsection{Estabilidad vertical de la atmósfera}

Los movimientos verticales en la atmósfera son de vital importancia, sobre todo en los fenómenos asociados a la convección. Por esta razón es imprescindible conocer la predisposición de la atmósfera a favorecer o inhibir estos movimientos a la hora de realizar una predicción.

El estudio de la estabilidad vertical se basa en considerar el hipotético desplazamiento de una parcela de aire o burbuja con el fin de analizar si ese desplazamiento es favorecido o no por la atmósfera circundante. A fin de simplificar el estudio se considera que la burbuja no se mezcla con el aire que la rodea y no lo perturba, no intercambia calor (el proceso es adiabático) y que las presiones, de burbuja y ambiente, son iguales. Este método trata de simular lo que puede ocurrir a una porción de aire que da lugar a la formación de un $\mathrm{Cu}$ mulonimbo, que se ha visto desplazada verticalmente en una zona de convergencia o debido a un ascenso orográfico. Inicialmente la burbuja forma parte de la masa de aire, con sus mismas propiedades de humedad y temperatura, pero se convierte en un sistema 
propio tan pronto como se produce su desplazamiento. Aunque los resultados que se obtienen con este método son exagerados y necesitan de correcciones para ser directamente aplicables sí que proporcionan conclusiones cualitativas correctas.

La estabilidad vertical se determina al comparar la densidad de la burbuja y de la atmósfera que la rodea. A igualdad de presiones el estudio de la estabilidad se traduce en comparar temperaturas: si la temperatura de la burbuja es mayor que su entorno su densidad será menor y ascenderá. Si se parte de una misma temperatura inicial entonces basta con comparar los gradiente de evolución de la burbuja y del entorno para determinar la estabilidad. De esta forma se tienen tres posibles estados:

- Estable: Si el gradiente atmosférico es mayor que el que sigue la burbuja. Los movimientos verticales son inhibidos.

- Inestable: Si el gradiente atmosférico es menor que la evolución de la burbuja. Se favorece que el movimiento continúe.

- Neutro: Si ambos gradientes son iguales se tiene un equilibrio neutro.

Los diagramas termodinámicos son la herramienta principal utilizada para evaluar la estabilidad atmos- férica. En ellos se representan los datos obtenidos de radiosondeos: humedad, temperatura y viento. De esta forma el gradiente vertical de la atmósfera se deduce de forma inmediata a partir de la pendiente que sigue la curva de temperatura (es más riguroso usar la temperatura virtual para realizar estas comparaciones, sobre todo para altos valores de temperatura y humedad donde los errores pueden ser apreciables). Los diagramas termodinámicos van provistos de una serie de isolíneas para facilitar estos análisis.

$\mathrm{Al}$ considerar que la burbuja no intercambia calor con el ambiente el gradiente que marcará su evolución será:

- Gradiente adiabático seco en caso de ser aire seco o húmedo sin saturar. En la práctica se dice que sigue la curva adiabática seca, o simplemente adiabática.

- Gradiente adiabático saturado en caso de haber saturación.

Debido a la diferencia que existe entre el gradiente adiabático seco y saturado es posible que la atmósfera sea estable si la burbuja no está saturada e inestable si lo está. Se dice entonces que la atmósfera es condicionalmente inestable. Visto en un diagrama termodinámico de tipo Skew-T (Figura 6.6).

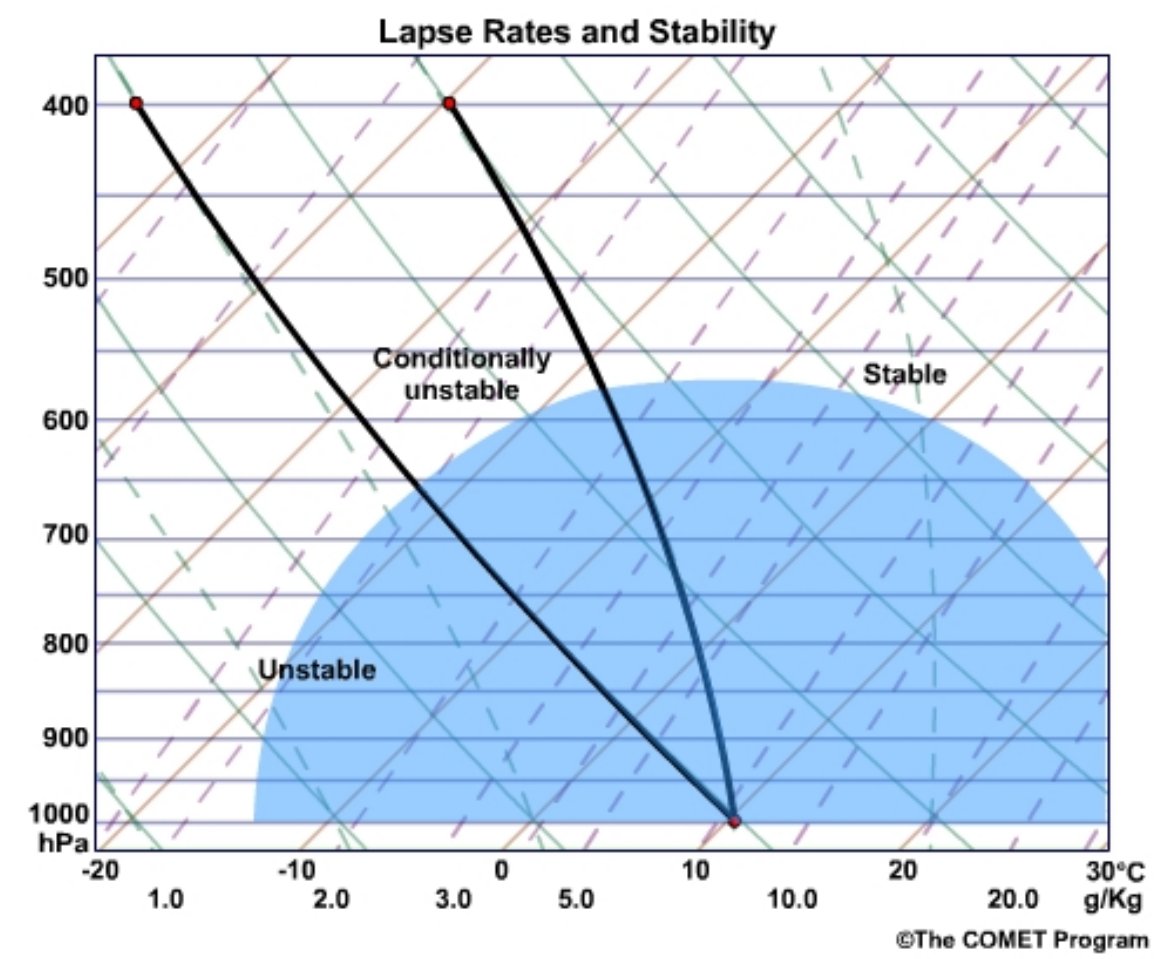

Figura 6.6: Condiciones de estabilidad según el gradiente en un diagrama Skew-T (The COMET Program, http: //www. meted. ucar. edu/mesoprim/skewt/, requiere registrarse). 


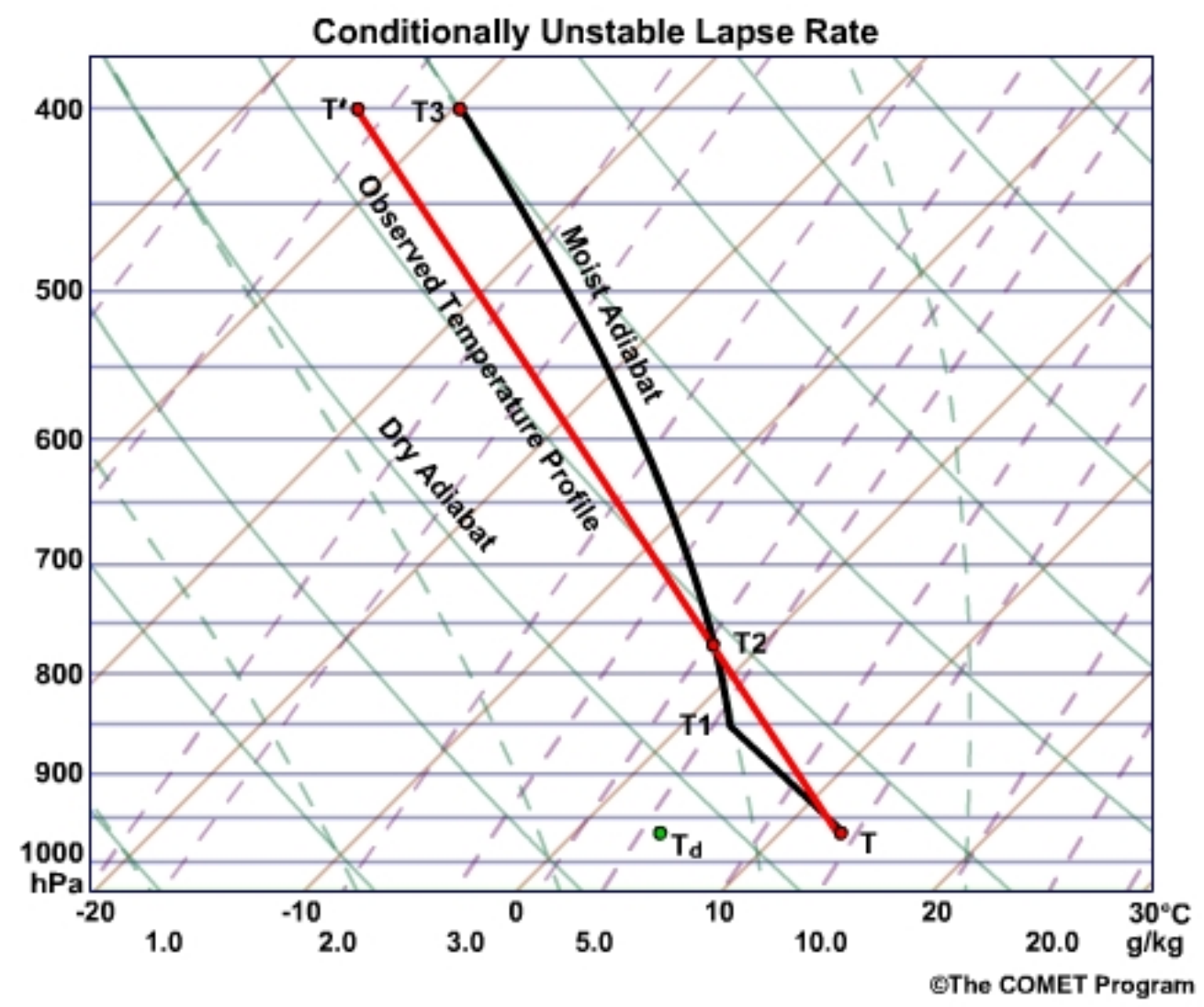

Figura 6.7: Atmósfera inestable para la burbuja que asciende desde 975 hPa y logra sobrepasar el NCL marcado por $T_{2}$ (The COMET Program, http: //www. meted. ucar. edu/mesoprim/skewt/, requiere registrarse).

La atmósfera será estable si su gradiente de temperatura es menor que el adiabático saturado. Será completamente inestable en caso que el gradiente sea superior al adiabático seco. Cuando la atmósfera es condicionalmente inestable es necesario conocer su contenido de humedad para poder evaluar la estabilidad. Para ello se realiza un desplazamiento inicial de la burbuja, siguiendo la adiabática seca hasta alcanzar la saturación. Este punto se conoce como nivel de condensación por ascenso NCA. A partir de ahí su evolución continua siguiendo la adiabática saturada. Si esta línea corta en algún momento el perfil de temperatura ambiente podrá evolución de forma libre. Este punto se conoce como nivel de convección libre NCL. Este es el caso ilustrado en la Figura 6.7, que muestra una atmósfera inestable para el desplazamiento de la burbuja considerada. Es importante remarcar que el análisis de la estabilidad condicional depende de la burbuja que se considere.

Se han explicado brevemente los fundamentos del análisis de estabilidad en base a las leyes de la termodinámica y bajo una serie de simplificaciones que permiten abordar el problema de forma más sencilla. A partir de aquí existe una bibliografía extensa para profundizar en la termodinámica en primer lugar y en el uso de diagramas como herramienta fundamental.

\subsection{Dinámica Atmosférica}

\subsubsection{Ecuaciones de la dinámica}

La dinámica atmosférica constituye un problema muy concreto de la dinámica de fluidos. La modelización del estado de la atmósfera se realiza a través de la resolución de las ecuaciones de NAVIER-STOKES [1] de un fluido en rotación sobre la superficie de la esfera terrestre, en un campo de gravedad, con forzamiento termodinámico externo solar, con multitud de componentes químicos, etc. Se trata en definitiva de un problema muy complejo que incluso tomando determinadas simplificaciones da muestra de la enorme dificultad que conlleva su resolución.

Las ecuaciones que gobiernan la dinámica de fluidos son no lineales, lo que significa que la evolución de un determinado valor no puede obtenerse numéricamente a partir una extrapolación de valores observados en un tiempo dado como por ejemplo puede ser la velocidad 
del fluido. Las soluciones de las ecuaciones no lineales presentan un comportamiento razonablemente lineal para intervalos de tiempo suficientemente cortos, sin embargo fuera de este rango temporal se hacen más grandes los términos no lineales lo que lleva a que el comportamiento de las soluciones parezca errático. Esto no quiere decir que no se trate de un problema determinista puesto que los valores en un tiempo futuro están, en teoría, completamente determinados por los valores en un instante dado. Las soluciones son altamente dependientes de sus propios valores y con una alta propagación de los errores numéricos. Pequeñas diferencias en las condiciones iniciales pueden dar lugar a soluciones considerablemente diferentes, lo cual fue detectado por primera vez a partir del estudio de las soluciones numéricas de la ecuación de convección [3].

Enfoques lagrangiano y euleriano de la dinámica. La descripción de la evolución de una burbuja de fluido se realiza en términos de la evolución que ésta sigue a lo largo de la trayectoria. La evolución de valores del estado de la burbuja (tales como la temperatura) se describe a lo largo de su movimiento, lo que se conoce como enfoque lagrangiano y es conceptualmente más natural que el enfoque euleriano que tiene en cuenta los valores del estado de un fluido para un punto espacial determinado. La comparación entre ambos valores tiene en cuenta el desplazamiento de la burbuja a través de un término de transporte o advección. Estos términos son inherentes a las derivadas temporales presentes en las ecuaciones de fluidos y son responsables entre otros del carácter no lineal de las mismas.

$$
\frac{d \vec{T}}{d t}=\frac{\partial \vec{T}}{\partial t}+\vec{v} \vec{\nabla} T
$$

\subsubsection{Aproximaciones}

Las ecuaciones de movimiento básicas de la atmósfera están asociadas a diferentes cantidades preservadas tendiendo en cuenta que la conservación tiene lugar a lo largo del movimiento de la partícula. El tratamiento de las ecuaciones en toda su complejidad constituye un problema inabordable, por lo que se realizan análisis de escala para realizar determinadas aproximaciones y eliminar ciertos términos de las ecuaciones que pueden ser despreciados debido a que toman valores suficientemente pequeños. La realización de una determinada simplificación está condicionada a la escala espacial y temporal que se pretende analizar [2].
Ecuaciones de EulER. Las ecuaciones (6.7) muestran relaciones asociadas a la conservación de momento, energía y masa tras efectuar un análisis de escala válido para la descripción de la dinámica atmosférica.

$$
\begin{array}{cl}
\frac{d \vec{v}}{d t}+f \vec{k} \times \vec{v}+\frac{1}{\rho} \vec{\nabla}_{z} p=0 & \text { momento horizontal } \\
\frac{d w}{d t}+g+\frac{1}{\rho} \partial_{z} p=0 & \text { momento vertical } \\
c_{p} \frac{d \ln T}{d t}-R \frac{d \ln p}{d t}=0 & \text { energía } \\
\frac{d \rho}{d t}+\rho \vec{\nabla} \vec{v}=0 & \text { masa }
\end{array}
$$

Un ejemplo de simplificación lo constituye la aproximación hidrostática, en la que se desprecia el término $\frac{d w}{d t}$ en la ecuación de momento vertical. En dicha aproximación la presión se identifica con el peso de la columna de aire en la vertical de un punto dado. Las escalas en las que la aproximación es válida son aquellas en las que no se tienen en cuenta los efectos de la convección, típicamente en distancias horizontales menores a $5 \mathrm{~km}$. Los modelos numéricos se dividen en hidrostáticos y no hidrostáticos según resuelvan o no estas escalas. En general las ecuaciones de la dinámica atmosférica suelen presentarse en términos de la coordenada vertical de presión en vez de altura, ello es debido a que según la aproximación hidrostática la presión es una variable monótonamente decreciente con la altura.

Si en latitudes medias consideramos únicamente la ecuación de momento horizontal y suponemos una situación en que el viento es constante a lo largo de la trayectoria $\frac{d \vec{v}}{d t}=0$ se obtiene una solución conocida como viento geostrófico en la que la fuerza del gradiente presión (o bárica) se equilibra con la fuerza de CORIOLIs (ficticia porque es consecuencia de la rotación terrestre) y el viento geostrófico viene dado únicamente por el campo de presión $\vec{v}_{g}=\vec{k} \times \frac{1}{\rho f} \vec{\nabla}_{z} p$. La dirección del viento es paralela a las isobaras dejando las bajas presiones a su lado izquierdo en el hemisferio norte. Además, la intensidad del viento es proporcional al gradiente de presión, por lo que las regiones de viento fuerte se corresponden con regiones en las que las isobaras está muy próximas entre sí. La relación del viento geostrófico a distintas alturas está dada por el gradiente térmico horizontal y la diferencia en distintos niveles de viento geostrófico se conoce como viento térmico. En las soluciones más realistas existen pequeñas componentes ageostróficas en las que el viento lleva aire de las zonas de mayor a menor presión. 


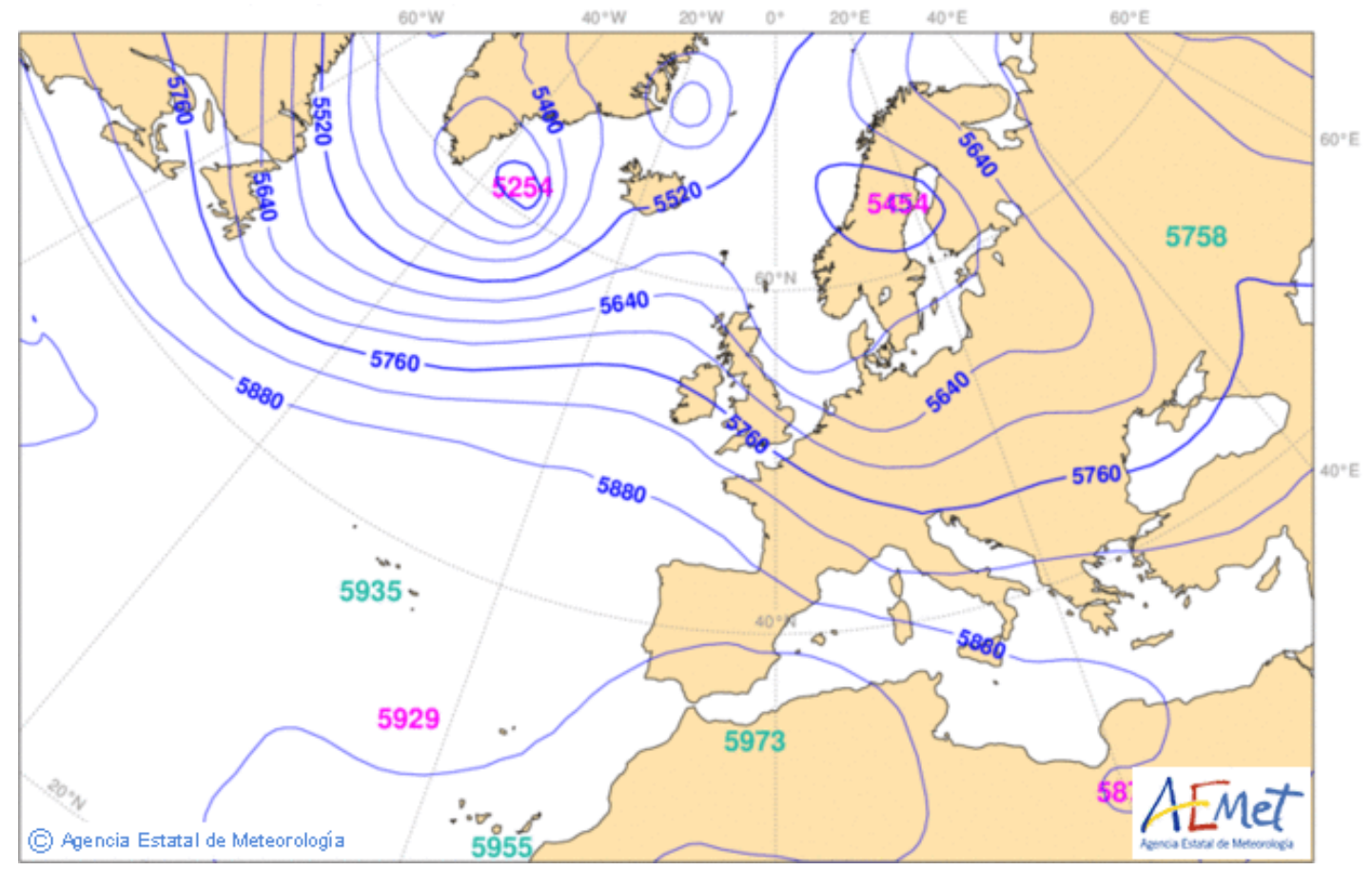

Figura 6.8: Mapa de altura geopotencial en 500 hPa (Agencia Estatal de Meteorología (AEMET)).

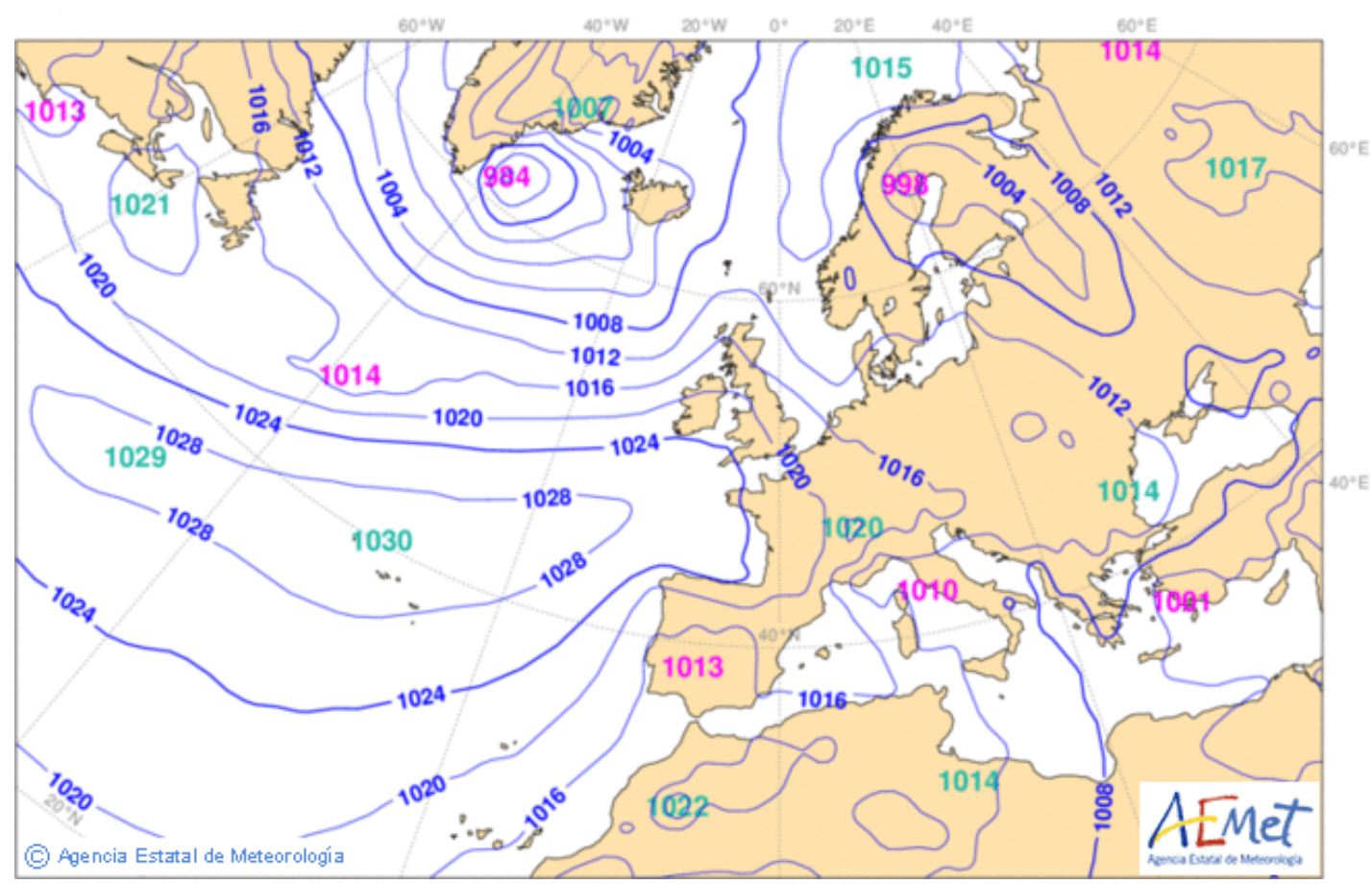

Figura 6.9: Mapa de presión en superficie (AEMET).

En las Figuras 6.8 y 6.9 pueden verse sendos mapas del modelo ECHRES con alcance de predicción de 60 horas para el 14 de julio a las 00 UTC. Mientras el mapa en superficie presenta líneas cerradas el mapa en altura muestra menor número de ellas, este es un hecho característico de ambos tipos de mapas. En la Figura (6.9) se observa una región de alta presión en superficie sobre la región de las Azores en la que el viento gira en sentido horario, sobre la región de Islandia hay una región de bajas presiones en las que el viento gira en sentido antihorario y al sur de la misma una región de viento fuerte de poniente. Este es el patrón característico de la región del Atlántico Norte. En el mapa en altura (Figura 6.8) lo que aparece son dorsales y vaguadas, relacionadas con perturbaciones de escala planetaria de interés en meteorología 
sinóptica (en las escalas de miles de kilómetros) y que tienen gran influencia sobre los fenómenos de superficie. Estas perturbaciones están descritas en términos de ondas de Rossby.

\subsubsection{Ondas}

Como se ha mencionado al inicio del presente capítulo, las soluciones de las ecuaciones no lineales presentan un comportamiento lineal en un determinado rango de tiempo. Por tanto, resulta de interés analizar el conjunto de ecuaciones linealizadas, es decir, aquellas en las que no se tienen en cuenta los términos no lineales (productos de dos, tres o más términos). Los términos lineales tienen asociados soluciones en términos de ondas, por lo que el conocimiento de las mismas es importante dado que representan estados de la atmósfera que podemos considerar como elementales. El estado de la atmósfera en un instante dado resultará ser una combinación de tales ondas. Los términos no lineales no introducen nuevos tipos de ondas, pero sí son responsables de interacciones entre ellas. En latitudes medias se dan tres tipos de ondas atmosféricas: acústicas, de gravedad y de RossBY.

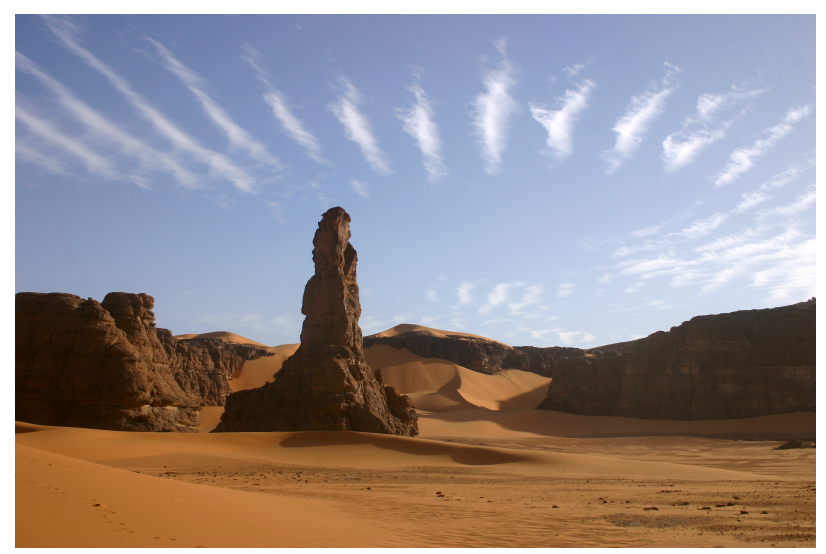

Figura 6.10: Onda de gravedad en la región de Tadrat, en Argelia, vemos que en los picos de las ondas se produce condensación (ver texto) (GFDL CC-BY-SA-3.0, https: // commons. wikimedia. org/wiki/File: Tadrart01. JPG).

Las ondas acústicas son ondas de compresión con velocidad de propagación de $300 \mathrm{~m} / \mathrm{s}$. Estas ondas no son de interés para el estudio de la atmósfera, por lo que es deseable que el conjunto de ecuaciones trate con alguna aproximación para filtrar este tipo de ondas. Tomando la aproximación hidrostática estas ondas desaparecen como solución de las ecuaciones.
Un tipo de ondas de sonido de propagación horizontal son las ondas de LAMB, que sólo se producen en erupciones volcánicas y explosiones nucleares atmosféricas y que carecen de interés meteorológico.

Las ondas de gravedad se generan como respuesta a desequilibrios en el campo de presión, como tiene lugar en una situación frontal o en caso de ascenso orográfico en situación estable (pudiendo generar nubes de tipo lenticular). Su fundamento físico es similar al de las ondas que se producen en la superficie del mar. Un análisis detallado de las ecuaciones linealizadas muestra que este tipo de ondas están emparentadas con las ondas de inercia. En realidad las dos son de un tipo más general que tiene a ambas como caso límite, las ondas de inercia-gravedad. Este tipo de ondas deben de ser controladas en la formulación de los modelos numéricos. Figura 6.10.

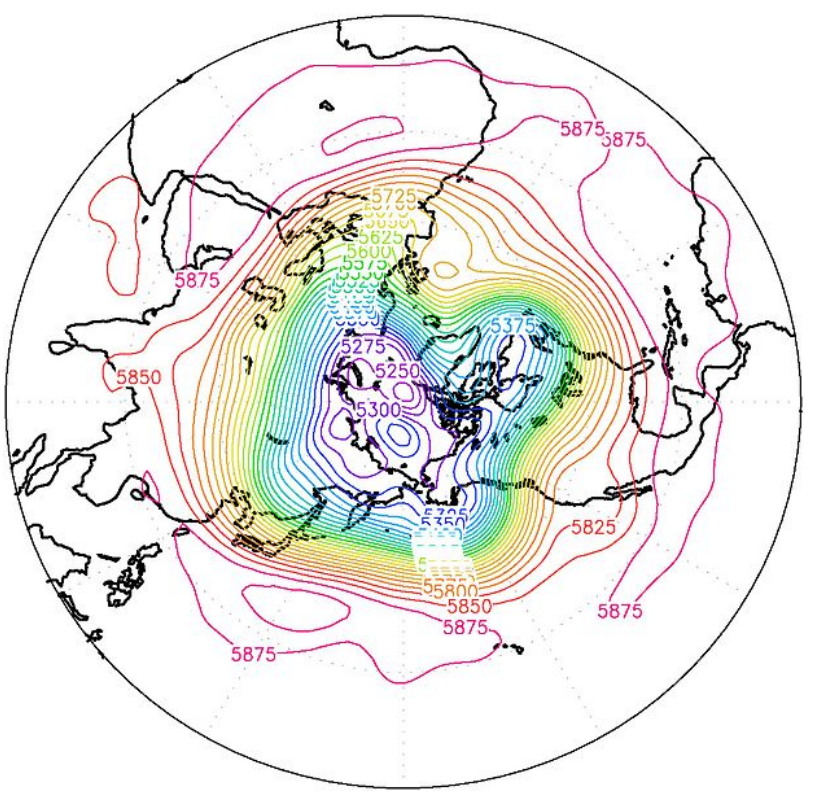

Figura 6.11: Onda de Rossby con número de onda 4, mapa de altura geopotencial en $500 \mathrm{hPa}$ promediada entre el 9 y el 21 de octubre de 2010 (ver texto) (CC BYSA 4.0, https:// commons. wikimedia. org/wiki/File: $500 \mathrm{mb}$ geopotential_height_wavenumber_4_Rossby wave_(planetary_waves)_pattern._October_9-21, _2010_daily_average. jpg).

Las ondas de RossBy son ondas producidas en un fluido en rotación debido a la conservación de la vorticidad potencial que tiene en cuenta tanto la vorticidad absoluta, suma de la vorticidad terrestre $f$ y de la vorticidad relativa del fluido con respecto a la tierra, como el espesor de la atmósfera.

El parámetro de CORIOLIS varía latitudinalmente por lo que un desplazamiento de aire latitudinal induce en 
el mismo vorticidad relativa. Además, por la dependencia con el espesor de la atmósfera, cuando el aire pasa por encima de una cadena montañosa se induce también vorticidad relativa. Tanto las Montañas Rocosas como los Andes, por su configuración norte-sur y teniendo en cuenta los vientos predominantes, son zonas de generación de ondas de Rossby de escala planetaria, que son las ondas de mayor interés meteorológico puesto que son responsables de la generación de dorsales y vaguadas de presión en altura y causantes de la generación de frentes que veremos más adelante. Figura 6.11.

\subsubsection{Circulación General de la Atmósfera}

El calentamiento de la superficie terrestre por la acción de la radiación solar depende de la latitud. En las regiones tropicales el azimut solar llega a ser perpendicular mientras que en las polares el azimut resulta siempre pequeño. Este calentamiento desigual da lugar como consecuencia a una transferencia de aire cálido de las zonas ecuatoriales a las polares. En un planeta en el que no hubiese rotación habría una única célula por cada hemisferio que llevase el aire cálido del ecuador a los polos en altura.

El efecto de la rotación terrestre produce, a través del término de CORIOLIS (6.7), que los vientos se desvíen hacia la derecha en el hemisferio norte y hacia la izquierda en el sur. Además, el efecto de la rotación terrestre rompe la célula hemisférica en tres, habiendo dos células directas: HADLEY (tropical) y polar, y una célula inversa: FERREL (en latitudes medias).

La separación de las células de HADLEY en ambos hemisferios está constituida por la zona de convergencia intertropical también conocida como de calmas ecuatoriales. Cada célula tiene asociada una masa de aire: cálida, templada o fría.

A su vez, el efecto de CORIOLIS para cada célula es responsable de los vientos predominantes: vientos del este en zonas polares y tropicales (alisios) y vientos de poniente en latitudes medias Figura 6.12. La separación entre la célula de FERREL y la célula polar está constituida por una región con fuerte gradiente térmico conocida como frente polar, que por el efecto del viento térmico da lugar un viento fuerte en altura ( $\mathrm{co}$ rriente en chorro o jet stream). El mismo fenómeno tiene lugar entre la célula de HADLEY y la célula de FERREL.

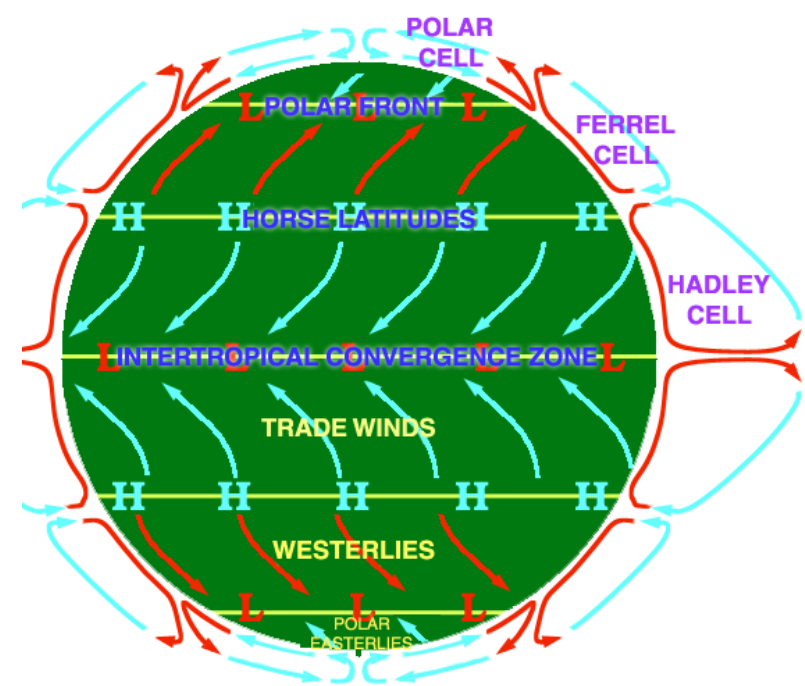

Figura 6.12: Diagrama representativo de la circulación general de la atmósfera con las células de HADLEY, FERREL y polar junto el régimen de vientos predominantes (ver texto) (CC BY-SA 3.0, https: // commons. wikimedia. org/ wiki/File: AtmosphCirc2. png).

\subsubsection{Frentes}

Un frente constituye una región de separación entre dos masas de aire con diferentes temperaturas. Este término fue acuñado por la escuela noruega de BERGEN como analogía con los frentes de guerra, ya que los primeros estudios tuvieron lugar durante la Primera Guerra Mundial. El modelo de formación de un ciclón extratropical establece que a partir de una perturbación del frente polar estacionario (producida por ondas de RosSBY) se generan dos regiones, una en las que avanza la masa de aire cálido (frente cálido) y otra en la que avanza el aire fío (frente frío). El frente frío avanza más rápidamente que el frente cálido llegando a alcanzar a este último formado un frente ocluido. En la Figura 6.13 puede verse el modelo conceptual de ciclogénesis de SHAPIRO-KEYSER similar al de la escuela noruega salvo por el hecho que permite la separación entre el frente frío y el frente cálido. El aire frío es más denso que el aire cálido por lo que en el caso de frente frío es el aire frío el que permanece pegado al suelo empujando al aire cálido por encima, como consecuencia se producen cumulus congestus y posteriormente cumulonimbus. En el caso del frente cálido, el avance del aire cálido tiene lugar por encima del aire frío con una pendiente bastante suave: un observador que contemple el avance de un frente cálido verá una secuencia de nubes asociadas primeramente cirrostratus, posteriormente altostratus y finalmente nimbostratus. 


\section{The evolution - Shapiro Keyser Cyclone}

From the modeling and observational studies,

this is the oceanic cyclone evolution Shapiro and Keyser T-BONE proposed. Many of the cyclones studied were off the U.S.

East Coast across our OFF waters.

Hit the return key or down arrow to continue... FRONTAL FRACTÜRE The uv vo omp lifies... the wo $\mathrm{mm}$ fromt buills seross the devoloping bw centor... I think us should cont inuoto useoceluded fromt

OPEN WAVE

Similerto the Fo rugerions the wo shoul conthuoto uso occuded fromt

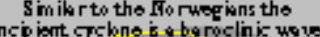
inbober for this bertion wo the center.
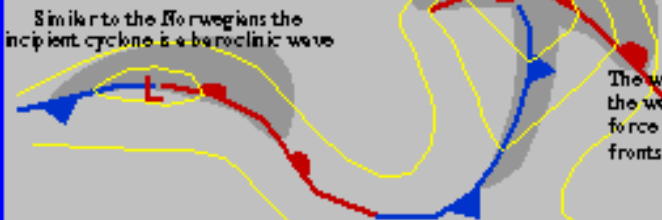
Tho wove comtinues to smp liff... fho (now) oceluded pertion of

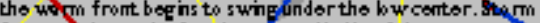

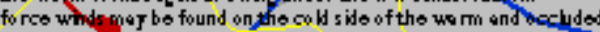
fromts.

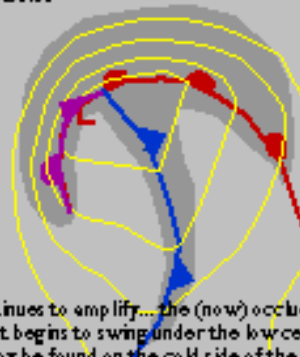

As the oceluded fromt encireles the bur centor o pothet of bw bvol uo $\mathrm{mm}$ a ir becomes entrapped over fho bw thus the sectus inn). Convection maj dovelop in thi us (m poctet is topse retes becomequito unstablo. In vorg dop crebnes uurricono fo ree winds maj bo found on the fold side of the tuded fromt.

MATURE

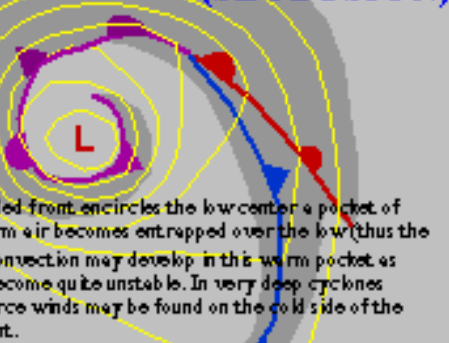

The temp grod iert o bng the $\mathrm{col}$ i fromt south of the wo rm frent becomer diffuse, thus the $\mathrm{col}$ W fromt froctures in this region and is $d$ rown 45 o frecture.
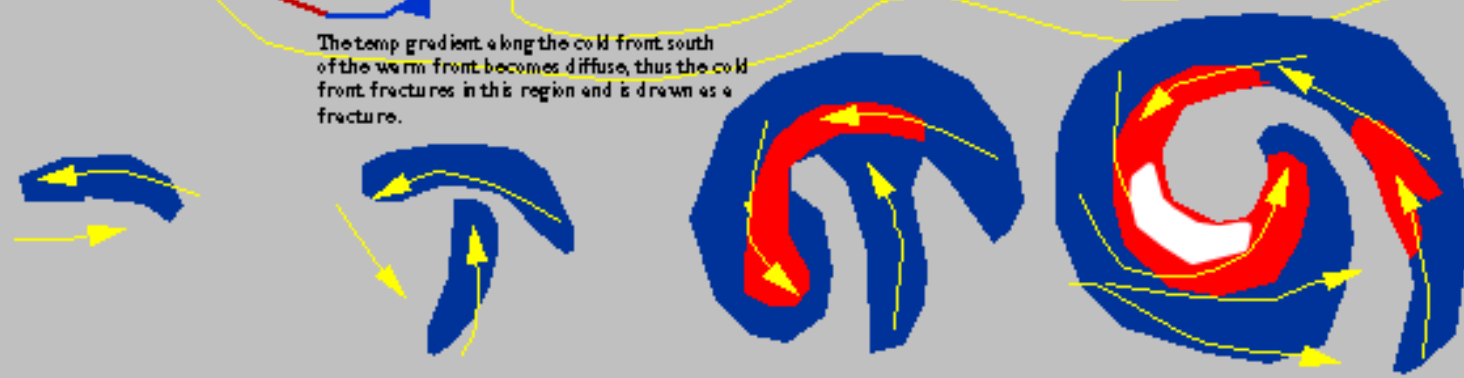

GALE WINDS

\section{STORM WINDS}

\section{HURRICANE FORCE WINDS}

Figura 6.13: Ciclo de vida de ciclón extratropical según el modelo de SHAPIRO-KeYSER (National Oceanic and Atmospheric Administration (NOAA), dominio público, https: // commons. wikimedia. org/wiki/File: Shapiro-Keyser_Cyclone. png). 


\subsection{Referencias}

[1] BAtchelor, G. K. (George Keith). An introduction to fluid dynamics. Cambridge University Press, 1999, página 615. ISBN: 9780521663960 (citado en página 77).

[2] Holton, James R. y HaKim, Gregory J. An introduction to dynamic meteorology.
Volumen 88. Academic press, 2012. ISBN: 0123848679 (citado en página 78).

[3] Lorenz, Edward N. "Deterministic Nonperiodic Flow". En: Journal of the Atmospheric Sciences 20.2 (mar. de 1963), páginas 130-141. ISSN: 0022-4928. DOI: 10 . $1175 / 1520-0469$ (1963) 020<0130: DNF> 2.0.CO;2 (citado en página 78). 